\title{
Sharp inequalities for the rates of convergence of the iterates of some operators which preserve the constants
}

\author{
Marius Mihai Birou
}

Dedicated to Professor Gheorghe Coman on the occasion of his 85th anniversary.

\begin{abstract}
In this paper we give estimates for the rates of convergence for the iterates of some positive linear operators which preserve only the constants. We obtain sharp inequalities when we use both continuous functions and differentiable functions. We present some optimal results for the Cesaro, Stancu and Schurer operators.
\end{abstract}

Mathematics Subject Classification (2010): 41A36, 41A25.

Keywords: Positive linear operators, iterates, convergence, sharp inequalities.

\section{Introduction}

Starting with the articles [9] and [8] of R.P. Kelisky, T.J. Rivlin and respectively S. Karlin, Z. Ziegler, the iterates of the positive linear operators were intensively studied.

The convergence of the sequence of the iterates of some positive linear operators which preserve only the constants was proved in [3], [14], [7], [13], [15], [4], [5], [6], [2].

On the other hand, estimations of the rates of convergence for the iterates of some positive operators preserving the constants were given in [10] using moduli of smoothness. In [1] the authors got sharp inequalities for the iterates of the Bernstein operators. In [12] the author obtained an estimate of the convergence rate for the iterations of linear and positive operators that reproduce linear functions in the case of differentiable functions.

In this note we obtain inequalities for the rates of convergence of the iterates of some positive linear operators $L: C[a, b] \rightarrow C[a, b]$ which preserve only the constants and have the interpolation point $x=a$ or $x=b$. In Section 2 we get these estimations both for continuous functions (using moduli of smoothness and divided difference) and 
for differentiable functions. The inequalities (2.1), (2.5), (2.6), (2.8), (2.9) and (2.12) are sharp in sense that we get equality if we take $f=e_{1}$. In Section 3 we determine the best constants in some inequalities involving the iterates of Cesaro, Stancu and Schurer operators.

Throughout the paper we use the following notations and definitions:

- the the monomial functions: $e_{i}:[a, b] \rightarrow \mathbb{R}, e_{i}(x)=x^{i}, i=0,1, \ldots$;

- the first and and the second moduli of smootness of the functiom $f \in C[a, b]$ :

$$
\omega_{1}(f, \delta)=\sup \{f(x+h)-f(x): x, x+h \in[a, b], 0 \leq h \leq \delta\},
$$

and respectively

$$
\omega_{2}(f, \delta)=\sup \{f(x+h)-2 f(x)+f(x-h): x, x \pm h \in[a, b], 0 \leq h \leq \delta\},
$$

where $\delta \geq 0$,

- the divided difference of the function $f \in C[a, b]$ on the distinct points $x_{1}, x_{2} \in$ $[a, b]$ :

$$
\left[x_{1}, x_{2} ; f\right]=\frac{f\left(x_{2}\right)-f\left(x_{1}\right)}{x_{2}-x_{1}} .
$$

\section{Main results}

Theorem 2.1. Let $L: C[a, b] \rightarrow C[a, b]$ be a positive linear operator which preserves only the constants and has interpolation point $x=a$. If

$$
L^{k} e_{1}(x)>a, x \in(a, b],
$$

then we have, for every $f \in C[a, b]$ and $x \in[a, b]$,

$$
\left|L^{k} f(x)-f(a)\right| \leq \frac{4}{b-a} \lambda_{k}(x) \omega_{1}\left(f, \lambda_{k}(x)\right)+3 \omega_{2}\left(f, \lambda_{k}(x)\right),
$$

where

$$
\lambda_{k}(x)=\frac{1}{2} \sqrt{(b-a)\left(L^{k} e_{1}(x)-a\right)} .
$$

Proof. Let $f \in C[a, b]$ and $0<\delta \leq(b-a) / 2$. If $F$ is a positive linear functional on $C[a, b]$, then from the optimal result of Păltănea [11] we have:

$$
\begin{gathered}
|f(x)-F(f)| \leq f(x)\left|F\left(e_{0}\right)-1\right|+\frac{1}{\delta}\left|F\left(e_{1}-x e_{0}\right)\right| \omega_{1}(f, \delta) \\
+\left(F\left(e_{0}\right)+\frac{1}{2 \delta^{2}} F\left(e_{1}-x e_{0}\right)^{2}\right) \omega_{2}(f, \delta), x \in[a, b] .
\end{gathered}
$$

Taking $F(f)=f(a)$ we get

$$
\begin{aligned}
|f-f(a)| & \leq \frac{e_{1}-a e_{0}}{\delta} \omega_{1}(f, \delta)+\left(e_{0}+\frac{\left(e_{1}-a e_{0}\right)^{2}}{2 \delta^{2}}\right) \omega_{2}(f, \delta) \\
& \leq \frac{e_{1}-a e_{0}}{\delta} \omega_{1}(f, \delta)+\left(e_{0}+\frac{(b-a)\left(e_{1}-a e_{0}\right)}{2 \delta^{2}}\right) \omega_{2}(f, \delta) .
\end{aligned}
$$


Since $L$ preserves the constant functions, it follows that

$$
\left|L^{k} f-f(a)\right| \leq \frac{1}{\delta}\left(L^{k} e_{1}-a e_{0}\right) \omega_{1}(f, \delta)+\left(e_{0}+\frac{(b-a)\left(L^{k} e_{1}-a e_{0}\right)}{2 \delta^{2}}\right) \omega_{2}(f, \delta) .
$$

If we take in $(2.4)$

$$
\delta=\lambda_{k}(x), x \in(a, b],
$$

where $\lambda_{k}$ is given by (2.2) we get that (2.1) holds for $x \in(a, b]$.

For $x=a$, due the interpolation property of $L$, we have $L^{k} f(a)=f(a)$. Therefore $(2.1)$ is also true for $x=a$. This completes the proof.

Theorem 2.2. Let $L: C[a, b] \rightarrow C[a, b]$ be a positive linear operator which preserves only the constants and has interpolation point $x=b$. If

$$
L^{k} e_{1}(x)<b, x \in[a, b),
$$

then we have, for every $f \in C[a, b]$ and $x \in[a, b]$,

$$
\left|L^{k} f(x)-f(b)\right| \leq \frac{4}{b-a} \mu_{k}(x) \omega_{1}\left(f, \mu_{k}(x)\right)+3 \omega_{2}\left(f, \mu_{k}(x)\right),
$$

where

$$
\mu_{k}(x)=\frac{1}{2} \sqrt{(b-a)\left(b-L^{k} e_{1}(x)\right)} .
$$

Proof. Taking $F(f)=f(b)$ in $(2.3)$ we get

$$
\begin{aligned}
|f-f(b)| & \leq \frac{b e_{0}-e_{1}}{\delta} \omega_{1}(f, \delta)+\left(e_{0}+\frac{\left(b e_{0}-e_{1}\right)^{2}}{2 \delta^{2}}\right) \omega_{2}(f, \delta) \\
& \leq \frac{b e_{0}-e_{1}}{\delta} \omega_{1}(f, \delta)+\left(e_{0}+\frac{(b-a)\left(b e_{0}-e_{1}\right)}{2 \delta^{2}}\right) \omega_{2}(f, \delta) .
\end{aligned}
$$

The conclusion follows analogous as in Theorem 2.1.

Theorem 2.3. Let $L: C[a, b] \rightarrow C[a, b]$ be a positive linear operator which preserves constants and has the interpolation point $x=a$. Then, for every $f \in C[a, b]$ and $x \in[a, b]$ we have

$$
m_{a}\left(L^{k}\left(e_{1}\right)(x)-a\right) \leq L^{k}(f)(x)-f(a) \leq M_{a}\left(L^{k}\left(e_{1}\right)(x)-a\right),
$$

where $m_{a}, M_{a} \in \mathbb{R}$ such that $m_{a} \leq[a, t ; f] \leq M_{a}$ when $t \in(a, b]$.

Proof. We have

$$
f(x)-f(a)=\left\{\begin{array}{cc}
{[a, x ; f](x-a),} & x \in(a, b] \\
0, & x=a
\end{array}\right.
$$

It follows

$$
m_{a}\left(e_{1}-a\right) \leq f-f(a) \leq M_{a}\left(e_{1}-a\right) .
$$

Applying $k$ times the operator $L$ on (2.7) we get the conclusion. 
Remark 2.4. From Theorem 2.3 we get the following criterion for the convergence of the iterates (see also [5, Corolar 2]): if $L: C[a, b] \rightarrow C[a, b]$ is a positive linear operator which preserves the constants, has the interpolation point $x=a$ and satisfies the condition

$$
\lim _{k \rightarrow \infty} L^{k} e_{1}=a, \text { uniformly on }[a, b]
$$

then for every $f \in C[a, b]$ we have

$$
\lim _{k \rightarrow \infty} L^{k} f=f(a) \text {, uniformly on }[a, b] .
$$

Theorem 2.5. Let $L: C[a, b] \rightarrow C[a, b]$ be a positive linear operator which preserves constants and has the interpolation point $x=b$. Then, for every $f \in C[a, b]$ and $x \in[a, b]$ we have

$$
m_{b}\left(b-L^{k}\left(e_{1}\right)(x)\right) \leq f(b)-L^{k}(f)(x) \leq M_{b}\left(b-L^{k}\left(e_{1}\right)(x)\right),
$$

where $m_{b}, M_{b} \in \mathbb{R}$ such that $m_{b} \leq[t, b ; f] \leq M_{b}$ for every $t \in[a, b)$.

The proof follows analogous with that of Theorem 2.3 using the formula

$$
f(b)-f(x)=\left\{\begin{array}{cc}
{[x, b ; f](b-x),} & x \in[a, b) \\
0, & x=b
\end{array}\right.
$$

Remark 2.6. From Theorem 2.5 we get the following criterion for the convergence of the iterates: if $L: C[a, b] \rightarrow C[a, b]$ is a positive linear operator which preserves the constants, has the interpolation point $x=b$ and satisfies the condition

$$
\lim _{k \rightarrow \infty} L^{k} e_{1}=b \text {, uniformly on }[a, b],
$$

then for every $f \in C[a, b]$ we have

$$
\lim _{k \rightarrow \infty} L^{k} f=f(b), \text { uniformly on }[a, b] \text {. }
$$

Theorem 2.7. Let $L: C[a, b] \rightarrow C[a, b]$ be a positive linear operator which preserves constants and has the interpolation point $x=a$. Then, for every $f \in C^{1}[a, b]$ and $x \in[a, b]$ we have

$$
m^{\prime}\left(L^{k}\left(e_{1}\right)(x)-a\right) \leq L^{k}(f)(x)-f(a) \leq M^{\prime}\left(L^{k}\left(e_{1}\right)(x)-a\right),
$$

where $m^{\prime}, M^{\prime} \in \mathbb{R}$ such that $m^{\prime} \leq f^{\prime}(t) \leq M^{\prime}, t \in[a, b]$ and

$$
\left|L^{k}(f)(x)-f(a)\right| \leq \overline{M^{\prime}}\left(L^{k}\left(e_{1}\right)(x)-a\right),
$$

where $\overline{M^{\prime}}=\max _{t \in[a, b]}\left|f^{\prime}(t)\right|$.

Proof. If $x \in(a, b]$, then using the mean value theorem it follows that there exists $\xi \in(a, x)$ such that

$$
f(x)-f(a)=(x-a) f^{\prime}(\xi) .
$$

If $x=a$ the formula (2.10) also holds for every $\xi \in[a, b]$.

Therefore

$$
m^{\prime}\left(e_{1}-a\right) \leq f-f(a) \leq M^{\prime}\left(e_{1}-a\right) .
$$

Applying $k$ times the operator $L$ on (2.11) we get (2.9). The proof is ended. 
Theorem 2.8. Let $L: C[a, b] \rightarrow C[a, b]$ be a positive linear operator which preserves constants and has the interpolation point $x=b$. Then, for every $f \in C^{1}[a, b]$ and $x \in[a, b]$ we have

$$
m^{\prime}\left(b-L^{k}\left(e_{1}\right)(x)\right) \leq f(b)-L^{k}(f)(x) \leq M^{\prime}\left(b-L^{k}\left(e_{1}\right)(x)\right),
$$

where $m^{\prime}, M^{\prime} \in \mathbb{R}$ such that $m^{\prime} \leq f^{\prime}(t) \leq M^{\prime}, t \in[a, b]$ and

$$
\left|L^{k}(f)(x)-f(b)\right| \leq \overline{M^{\prime}}\left(b-L^{k}\left(e_{1}\right)(x)\right),
$$

where $\overline{M^{\prime}}=\max _{t \in[a, b]}\left|f^{\prime}(t)\right|$.

The proof follows analogous with that of Theorem 2.7 using the mean value theorem:

$$
f(b)-f(x)=(b-x) f^{\prime}(\xi), \xi \in(a, b) .
$$

\section{Applications} stants:

We consider the following positive linear operators which preserve only the con-

- Cesaro operator

$$
C: C[0,1] \rightarrow C[0,1], C(f)(x)=\left\{\begin{array}{cl}
f(0), & x=0 \\
\frac{1}{x} \int_{0}^{x} f(t) d t, & x>0
\end{array}, x \in[0,1]\right.
$$

- Bernstein-Stancu operators (see [16])

$$
\begin{gathered}
S_{n, \alpha}: C[0,1] \rightarrow C[0,1], S_{n, \alpha}(f)(x)=\sum_{i=0}^{n}\left(\begin{array}{c}
n \\
i
\end{array}\right) x^{i}(1-x)^{n-i} f\left(\frac{i+\alpha}{n+\alpha}\right), \\
x \in[0,1], n=0,1, \ldots, \alpha>0,
\end{gathered}
$$

and

$$
\begin{gathered}
S_{n, \beta}: C[0,1] \rightarrow C[0,1], S_{n, \beta}(f)(x)=\sum_{i=0}^{n}\left(\begin{array}{c}
n \\
i
\end{array}\right) x^{i}(1-x)^{n-i} f\left(\frac{i}{n+\beta}\right), \\
x \in[0,1], n=0,1, \ldots, \beta>0,
\end{gathered}
$$

- Schurer operator

$$
\begin{gathered}
S_{n, p}: C[0,1] \rightarrow C[0,1], S_{n, p}(f)(x)=\sum_{i=0}^{n-p}\left(\begin{array}{c}
n-p \\
i
\end{array}\right) x^{i}(1-x)^{n-p-i} f\left(\frac{i}{n}\right), \\
x \in[0,1], n, p \in \mathbb{N}, n \geq p .
\end{gathered}
$$

The operators $C, S_{n, \beta}, S_{n, p}$ have the interpolation point $x=0$ while the operator $S_{n, \alpha}$ interpolates the continuous functions at $x=1$. For every $k \geq 0$ we have by induction (see also [5] for the operators $C, S_{n, \beta}, S_{n, p}$ ):

$$
C^{k} e_{1}=\frac{1}{2^{k}} e_{1}, S_{n, \alpha}^{k} e_{1}=\left(\frac{n}{n+\beta}\right)^{k} e_{1}, S_{n, p}^{k} e_{1}=\left(\frac{n-p}{n}\right)^{k} e_{1},
$$




$$
S_{n, \alpha}^{k} e_{1}=e_{0}+\left(\frac{n}{n+\alpha}\right)^{k}\left(e_{1}-e_{0}\right) .
$$

From Theorem 2.1 and Theorem 2.2 we have:

Theorem 3.1. For every $f \in C[0,1]$ and $x \in[0,1]$ we have:

1.

2.

$$
\left|C^{k} f(x)-f(0)\right| \leq 2 \sqrt{\frac{x}{2^{k}}} \cdot \omega_{1}\left(f, \frac{1}{2} \sqrt{\frac{x}{2^{k}}}\right)+3 \omega_{2}\left(f, \frac{1}{2} \sqrt{\frac{x}{2^{k}}}\right)
$$

$$
\begin{gathered}
\left|S_{n, \alpha}^{k} f(x)-f(0)\right| \leq \\
2 \sqrt{\left(\frac{n}{n+\beta}\right)^{k} x} \cdot \omega_{1}\left(f, \frac{1}{2} \sqrt{\left(\frac{n}{n+\beta}\right)^{k}} x\right)+3 \omega_{2}\left(f, \frac{1}{2} \sqrt{\left(\frac{n}{n+\beta}\right)^{k}} x\right),
\end{gathered}
$$

3.

$$
\begin{gathered}
\left|S_{n, p}^{k} f(x)-f(0)\right| \leq \\
2 \sqrt{\left(\frac{n-p}{n}\right)^{k} x} \cdot \omega_{1}\left(f, \frac{1}{2} \sqrt{\left(\frac{n-p}{n}\right)^{k} x}\right)+3 \omega_{2}\left(f, \frac{1}{2} \sqrt{\left(\frac{n-p}{n}\right)^{k}} x\right),
\end{gathered}
$$

4.

$$
\begin{gathered}
\left|S_{n, \beta}^{k} f(x)-f(1)\right| \leq \\
2 \sqrt{\left(\frac{n}{n+\alpha}\right)^{k} x} \cdot \omega_{1}\left(f, \frac{1}{2} \sqrt{\left(\frac{n}{n+\alpha}\right)^{k}} x\right)+3 \omega_{2}\left(f, \frac{1}{2} \sqrt{\left(\frac{n}{n+\alpha}\right)^{k}} x\right) .
\end{gathered}
$$

Using Theorem 2.3, Theorem 2.5 and respectively Theorem 2.7, Theorem 2.8 we get the following sharp estimates:

Theorem 3.2. Let $f \in C[0,1]$. If $m_{0}, M_{0}, m_{1}, M_{1} \in \mathbb{R}$ such that $m_{0} \leq[0, t ; f] \leq M_{0}$, $t \in(0,1]$ and $m_{1} \leq[t, 1 ; f] \leq M_{1}, t \in[0,1)$ then for every $k \geq 0$ we have:

1. $m_{0} c_{1}(k) e_{1} \leq C^{k}(f)-f(0) e_{0} \leq M_{0} c_{1}(k) e_{1}$, where $c_{1}(k)=\frac{1}{2^{k}}$,

2. $m_{0} c_{2}(k, n, \beta) e_{1} \leq S_{n, \beta}^{k}(f)-f(0) e_{0} \leq M_{0} c_{2}(k, n, \beta) e_{1}$, where

$$
c_{2}(k, n, \beta)=\left(\frac{n}{n+\beta}\right)^{k} \text {, }
$$

3. $m_{0} c_{3}(k, n, p) e_{1} \leq S_{n, p}^{k}(f)-f(0) e_{0} \leq M_{0} c_{3}(k, n, p) e_{1}$, where $c_{3}(k, n, p)=\left(\frac{n-p}{n}\right)^{k}$,

4. $m_{1} c_{4}(k, n, \alpha)\left(e_{0}-e_{1}\right) \leq f(1) e_{0}-S_{n, \alpha}^{k}(f) \leq M_{1} c_{4}(k, n, \alpha)\left(e_{0}-e_{1}\right)$, where $c_{4}(k, n, \alpha)=\left(\frac{n}{n+\alpha}\right)^{k}$.

Theorem 3.3. Let $f \in C^{1}[0,1]$. If $m^{\prime}, M^{\prime} \in \mathbb{R}$ such that $m^{\prime} \leq f^{\prime}(t) \leq M^{\prime}, t \in[0,1]$, then for every $k \geq 0$ we have:

1. $m^{\prime} c_{1}(k) e_{1} \leq C^{k}(f)-f(0) e_{0} \leq M^{\prime} c_{1}(k) e_{1}$

2. $m^{\prime} c_{2}(k, n, \beta) e_{1} \leq S_{n, \beta}^{k}(f)-f(0) e_{0} \leq M^{\prime} c_{2}(k, n, \beta) e_{1}$,

3. $m^{\prime} c_{3}(k, n, p) e_{1} \leq S_{n, p}^{k}(f)-f(0) e_{0} \leq M^{\prime} c_{3}(k, n, p) e_{1}$,

4. $m^{\prime} c_{4}(k, n, \alpha)\left(e_{0}-e_{1}\right) \leq f(1) e_{0}-S_{n, \alpha}^{k}(f) \leq M^{\prime} c_{4}(k, n, \alpha)\left(e_{0}-e_{1}\right)$,

where the constants $c_{1}(k), c_{2}(k, n, \beta), c_{3}(k, n, p), c_{4}(k, n, \alpha)$ are given in Theorem 3.2 . 
The constants $c_{1}(k), c_{2}(k, n, \beta), c_{3}(k, n, p), c_{4}(k, n, \alpha)$ in Theorem 3.2 and Theorem 3.3 are the best possible: for $f=e_{1}$ we get equality.

\section{References}

[1] Abel, U., Ivan, M., New representation of the remainder in the Bernstein approximation, J. Math. Anal. Appl., 381(2011), 952-956.

[2] Altomare, F., On some convergence criteria for nets of positive operators on continuous function spaces, J. Math. Anal. Appl., 398(2013), 542-552.

[3] Altomare, F., Campiti, M., Korovkin-Type Approximation Theory and Its Applications, vol. 17 of de Gruyter Studies in Mathematics, Walter de Gruyter, Berlin, Germany, 1994.

[4] Galaz Fontes, F., Solis, F.J., Iterating the Cesaro operators, Proc. Amer. Math. Soc., 136(2008), no. 6, 2147-2153.

[5] Gavrea, I., Ivan, M., The iterates of positive linear operators preserving constants, Appl. Math. Lett., 24(2011), 2068-2071.

[6] Gavrea, I., Ivan, M., Asymptotic Behaviour of the Iterates of Positive Linear Operators, Hindawi Publishing Corporation, Abstr. Appl. Anal., Volume 2011, Article ID 670509, 11 pages.

[7] Gonska, H., Pitul, P., Raşa, I., Over-iterates of Bernstein-Stancu operators, Calcolo, 44(2007), no. 2, 117-125.

[8] Karlin, S., Ziegler, Z., Iteration of positive approximation operators, J. Approx. Theory, 3(1970), 310-339.

[9] Kelisky, R.P., Rivlin, T.J., Iterates of Bernstein polynomials, Pacific J. Math., 21(1967), 511-520.

[10] Mahmudov, N.I., Asymptotic properties of iterates of certain positive linear operators, Math. Comput. Model., 57(2013), 1480-1488.

[11] Păltănea, R., Optimal estimates with moduli of continuity, Results Math., 32(1997), 318-331.

[12] Raşa, I., Asymptotic behaviour of iterates of positive linear operators, Jaen J. Approx., 1(2009), no. 2, 195-204.

[13] Raşa, I., $C_{0}$-Semigroups and iterates of positive linear operators: asymptotic behaviour, Rend. Circ. Mat. Palermo, 82(2010), no. 2, 123-142.

[14] Rus, I.A., Iterates of Stancu operators, via contraction principle, Stud. Univ. BabeşBolyai Math., 47(2002), no. 4, 101-104.

[15] Rus, I.A., Iterates of Stancu operators (via fixed point principles) revisited, Fixed Point Theory, 11(2010), no. 2, 369-374.

[16] Stancu, D.D., Asupra unei generalizări a polinoamelor lui Bernstein, Stud. Univ. BabeşBolyai Math., 14(1969), no. 2, 31-45.

Marius Mihai Birou

Technical University of Cluj Napoca, Department of Mathematics, 28, Memorandumului Street, 400114 Cluj-Napoca, Romania

e-mail: Marius.Birou@math.utcluj.ro 